\title{
FRACTAL DIMENSIONS AND SINGULARITIES OF THE WEIERSTRASS TYPE FUNCTIONS
}

\author{
TIAN-YOU HU AND KA-SING LAU
}

\begin{abstract}
A new type of fractal measures $\mathscr{K}^{s}, 1 \leq s \leq 2$, defined on the subsets of the graph of a continuous function is introduced. The $\mathscr{K}$-dimension defined by this measure is 'closer' to the Hausdorff dimension than the other fractal dimensions in recent literatures. For the Weierstrass type functions defined by $W(x)=\sum_{0}^{\infty} \lambda^{-\alpha i} g\left(\lambda^{i} x\right)$, where $\lambda>1,0<\alpha<1$, and $g$ is an almost periodic Lipschitz function of order greater than $\alpha$, it is shown that the $\mathscr{K}$-dimension of the graph of $W$ equals to $2-\alpha$, this conclusion is also equivalent to certain rate of the local oscillation of the function. Some problems on the ' knot' points and the nondifferentiability of $W$ are also discussed.
\end{abstract}

\section{INTRODUCTION}

It is easy to show that if $f \in \operatorname{Lip}(\alpha)$ (the class of Lipschitz functions of order $\alpha), 0<\alpha<1$, then the Hausdorff dimension of the graph of $f$, denoted by $\Gamma_{f}$, does not exceed $2-\alpha$. On the other hand, there is no satisfactory condition to estimate the lower bound of the dimension of $\Gamma_{f}$. In particular, it is an open question that for $\lambda>1,0<\alpha<1$, the Hausdorff dimension of the graph of the Weierstrass function

$$
W(x)=\sum_{i=1}^{\infty} \lambda^{-\alpha i} \sin \left(\lambda^{i} \pi x\right), \quad x \in \mathbb{R},
$$

(or more general, replace the sine function by a bounded differentiable almost periodic function) equals to $2-\alpha$. Recall that for $s \geq 0$ the Hausdorff measure $\mathscr{H}^{s}$ is defined on subsets of $\mathbb{R}^{n}$ by

$$
\mathscr{H}^{s}(E)=\lim _{\delta \rightarrow 0^{+}} \mathscr{H}_{\delta}^{s}(E),
$$

where

$$
\mathscr{H}_{\delta}^{s}(E)=\inf \left\{\sum_{i}\left(\operatorname{diam} U_{i}\right)^{s}: E \subseteq \bigcup_{i} U_{i}, \operatorname{diam} U_{i}<\delta\right\},
$$

$\left\{U_{i}\right\}_{1}^{\infty}$ are open subsets of $\mathbb{R}^{n}$. The Hausdorff dimension of $E$, denoted by $\mathscr{H}-\operatorname{dim} E$, is defined by $\mathscr{H}-\operatorname{dim} E=\inf \left\{s>0: \mathscr{H}^{s}(E)=0\right\}$.

Received by the editors March 20, 1990 and, in revised form, October 29, 1990.

1980 Mathematics Subject Classification (1985 Revision). Primary 28A12; Secondary 42A32.

Key words and phrases. Almost periodic functions, box dimension, Hausdorff dimension, knot points, nondifferentiability, Weierstrass type functions. 
There are many attempts in solving the problem stated in (1.1). Besicovitch and Ursell [1] gave a positive answer by modifying the term $\lambda^{-\alpha i}$ to $\lambda_{i}$ such that $\lambda_{i+1} / \lambda_{i}$ increases to $\infty$ and $\log \lambda_{i+1} / \log \lambda_{i} \rightarrow 1$. Kaplan, Mallet-Paret and Yorke [10] and Rezakhanlou [13] proved respectively that the box dimension and the packing dimension (see $\S 3$ ) of $\Gamma_{W}$ is $2-\alpha$. Mauldin and Williams [11] using a Cantor set argument concluded that the Hausdorff dimension of $\Gamma_{W}$ is greater than $2-\alpha-(c / \log \lambda)$, provided that $\lambda$ is large. More recently the authors [7] considered the replacement of $W$ by the Rademacher series

$$
R(x)=\sum_{i=1}^{\infty} 2^{-\alpha i} R_{i}(x), \quad x \in[0,1]
$$

where $R_{1}(x)=\operatorname{sign}(\sin 2 \pi x)$, and $R_{i}(x)=R_{1}\left(2^{i-1} x\right), i=1,2, \ldots$, is a sequence of Rademacher functions. They showed that if the distribution function $F$ of $R$ is absolutely continuous and $F^{\prime} \in L^{p}$ for some $p>1$, then the Hausdorff dimension of $\Gamma_{R}$ is $2-\alpha$. This is the case when $\alpha=1 / n, n=1,2, \ldots$. Furthermore the result is true for all $0<\alpha<1$ if a small perturbation of $2^{-\alpha i}$ in the sense of Kahane and Salem [9] is allowed [8]. The first result of the Rademacher series $R(x)$ was also obtained by Przytycki and Urbanski [12] by using a dynamics argument; they also proved a more striking result: there exist some values of $\alpha$ ( $2^{\alpha}$ is a Pisot-Vijayaraghavan number) so that the graph of $R(x)$ has Hausdorff dimension strictly less than $2-\alpha$.

The main purpose of the paper is twofold: First we introduce another type of measures $\mathscr{K}^{s}, 1 \leq s \leq 2$, on the subsets of the graph of a continuous function $f$, and define a new $\mathscr{K}$-dimension index. While the Hausdorff dimension is determined by the measure using arbitrary open covers, and the box dimension is corresponding to covers with balls of equal size, the new scheme is a mixture of these two. The importance of such consideration is that

(i) $\mathscr{K}^{s}$ is a genuine measure on the subsets of $\Gamma_{f}$;

(ii) With regard to the continuous curves, the $\mathscr{K}$-dimension is an improvement (closer to the Hausdorff dimension) of the box dimension and other dimensions in the literature (Theorems 3.1 and 3.2);

(iii) $\mathscr{K}^{s}\left(\Gamma_{f}\right)$ is closely related to different variational norms of functions on $\mathbb{R} ;$

(iv) It is a natural tool to deal with Lipschitz functions, especially for the Weierstrass type functions (Lemma 2.4 and $\S 4$ ).

Secondly, we study the local oscillation behavior of the Weierstrass type functions. For $\lambda>1,0<\alpha<\beta \leq 1$, let $g(x)$ defined on $\mathbb{R}$ be a bounded real-valued (Bohr) almost periodic Lipschitz function of order $\beta$, let $W$ be a Weierstrass type function defined by

$$
W(x)=\sum_{0}^{\infty} \lambda^{-\alpha i} g\left(\lambda^{i} x\right), \quad x \in \mathbb{R} .
$$

By substracting a constant, we can assume, without loss of generality, that $g(0)=0$. Let

$$
V(x)=\sum_{-\infty}^{\infty} \lambda^{-\alpha i} g\left(\lambda^{i} x\right), \quad x \in \mathbb{R}
$$


then the series converges for all $x, V$ is also known as the Weierstrass-Mandelbrot function. For any real-valued function $f$ on $\mathbb{R}, \varepsilon>0, x \in \mathbb{R}$ and any interval $I_{x}$ containing $x$, let

$$
E\left(f, \varepsilon, I_{x}\right)=\left\{y \in I_{x}:|f(y)-f(x)| /|y-x|^{\alpha}>\varepsilon, y \neq x\right\} .
$$

Our main result is Theorem 4.1. We prove that $V \not \equiv 0$ if and only if $\mathscr{K}$ $\operatorname{dim} \Gamma_{w}=2-\alpha$, which is also equivalent to that there exist positive numbers $\varepsilon, \eta$ and $c$ so that $\left|E\left(W, \varepsilon, I_{x}\right)\right| /\left|I_{x}\right| \geq c$ for every $x \in \mathbb{R}$ and every interval $I_{x}$ containing $x$ with $\left|I_{x}\right|<\eta$, where $|A|$ denotes the Lebesgue measure of $A$ for any Lebesgue measurable subset $A$ of $\mathbb{R}$. This improves the previous results of Hata [6], Kaplan et al. [10], and of Rezakhanlou [13].

The singularities of the Weierstrass function have been attracting much attention for a long time (see [5, 6, 10 and 12]). Recently Hata made another detail study and strengthened most of the previous results by using expressions similar to (1.4). By modifying the idea of the proof of Theorem 4.1, we can improve some of his results. Our proofs are especially simple since we make use of the 'relatively dense sets' of almost periodic functions and the auxiliary function $V(x)$ in (1.3) instead of his spectral approach.

The paper is organized as follows: In $\S 2$ we introduce the $\mathscr{K}^{s}$-measure and the notion of the $\mathscr{K}$-dimension. In $\S 3$ we compare the $\mathscr{K}$-dimension with the other dimension indices. We also observe that the $\mathscr{K}^{s}$-measure of the graph of $f$ is related to some variational norms of $f$. The main part is $\S 4$, which proves the equivalence between the fractal dimension and the local oscillation of $W$. In $\S 5$, we follow the idea used in $\S 4$, and improve a theorem of Hata on the ' $k$ not' points of the Weierstrass type functions. Some remarks of the theorems and comparisons of the conditions used by Hata and Kaplan et al. are also given.

Acknowledgments. The authors would like to express their gratitude to Professor T. A. Metzger and Professor W. B. Zeng for bringing to their attention some relevant literature. They also thank the referee for many helpful comments.

\section{A NEW DEFINITION OF DIMENSION}

Let $f$ be a continuous function defined on $[0,1]$ with graph $\Gamma_{f}$. For any open interval $I$ of $[0,1]$, let $q_{I}$ be the least number of open squares $I \times I^{\prime}$ whose union covers $f(I)$. It is clear that

$$
q_{I}-1 \leq \operatorname{osc}(f, I) /|I| \leq q_{I},
$$

where $\operatorname{osc}(f, I)$ denotes the oscillation of $f$ on the interval $I$. Let $s \geq 0$, and let $P$ be the natural projection from $\mathbb{R}^{2}$ to $\mathbb{R}$ defined by $P(x, y)=x$. For any subset $E$ of $\Gamma_{f}$ and arbitrary open cover $\mathscr{C}$ of $P(E)$, define

$$
\varphi^{s}(E, \mathscr{C})=\sum_{I \in \mathscr{C}} q_{I}|I|^{s},
$$

and

$$
\mathscr{K}^{s}(E)=\lim _{\delta \rightarrow 0^{+}} \inf _{|\mathscr{E}|<\delta} \varphi^{s}(E, \mathscr{C}),
$$

where $|\mathscr{C}|=\sup \{|I|: I \in \mathscr{C}\}$. A routine check shows that $\mathscr{K}^{s}$ is an outer measure on $\Gamma_{f}$. As usual we call a subset $E$ of $\Gamma_{f} \mathscr{K}^{s}$-measurable if $\mathscr{K}^{s}(A)=$ 
$\mathscr{K}^{s}(A \cap E)+\mathscr{K}^{s}\left(A \cap E^{c}\right)$, for all subsets $A$ of $\Gamma_{f} ; \mathscr{K}^{s}$ is then a measure on the $\sigma$-algebra of all $\mathscr{K}^{s}$-measurable subsets. It is also easy to check that $\mathscr{K}^{s}$ is a metric outer measure in the sense that $\mathscr{K}^{s}(E \cup F)=\mathscr{K}^{s}(E)+\mathscr{K}^{s}(F)$, whenever the distance in $\mathbb{R}^{2}$ between $E$ and $F$ is positive, hence it follows from an argument of Carathéodory that all the Borel subsets of $\Gamma_{f}$ are $\mathscr{K}^{s}$ measurable (see [4, pp. 5-6]).

We remark that $\mathscr{K}^{s}$ can be defined similarly on all subsets of $\mathbb{R}^{2}$, however, in such case, it would not be an outer measure any more.

We omit the simple proof for the following proposition which excludes the trivial cases of $s$.

Proposition 2.1. Let $f$ be a continuous function defined on $[0,1]$.

(i) If $0<s<1$, then $\mathscr{K}^{s}\left(\Gamma_{f}\right)=\infty$; and

(ii) If $s>2$, then $\mathscr{K}^{s}(E)=0$ for any subset $E$ of $\Gamma_{f}$.

The $\mathscr{K}^{s}$-measure of $\Gamma_{f}$ can be put into the following simpler form in connection with the variational norms which will be considered in the next section.

Proposition 2.2. Let $f$ be a continuous function defined on $[0,1]$, and let $1<$ $s \leq 2$, then

$$
\mathscr{K}^{s}\left(\Gamma_{f}\right)=\lim _{\delta \rightarrow 0^{+}} \inf _{|\Pi|<\delta} \sum_{\Pi} \operatorname{osc}\left(f, \Delta_{i}\right)\left|\Delta_{i}\right|^{s-1},
$$

where $\Pi=\left\{0=x_{0}<x_{1}<\cdots<x_{n}=1\right\}$ is a partition of $[0,1], \Delta_{i}=$ $\left[x_{i-1}, x_{i}\right)$, and $|\Pi|=\max \left|\Delta_{i}\right|$.

Proof. We first show that the left-hand side of (2.2) is not greater than the righthand side. Let $M=\operatorname{osc}(f,[0,1])$. For $\delta>0$, let $\Pi=\left\{0=x_{0}<x_{1}<\cdots<\right.$ $\left.x_{n}=1\right\}$ be a partition of $[0,1]$ with $\sum\left|\Delta_{i}\right|^{s}<\delta$. Notice that such a partition can be found because $s>1$. Let $\eta>0$ be such that $n M(2 \eta)^{s-1}+n(2 \eta)^{s}<\delta$. Let $\mathscr{C}$ be the open cover of $[0,1]$ consisting of $\Delta_{i}^{\circ}$, the interior of $\Delta_{i}$, and $\Delta_{i}^{\prime}=\left(x_{i}-\eta, x_{i}+\eta\right), i=1, \ldots, n$. Then by $(2.1)$,

$$
\begin{aligned}
\varphi^{s}([0,1], \mathscr{C}) & =\sum_{i=1}^{n} q_{\Delta_{i}^{\circ}}\left|\Delta_{i}\right|^{s}+\sum_{i=1}^{n} q_{\Delta_{i}^{\prime}}^{s}\left|\Delta_{i}^{\prime}\right|^{s} \\
& \leq \sum_{i=1}^{n}\left(1+\operatorname{osc}\left(f, \Delta_{i}\right) /\left|\Delta_{i}\right|\right)\left|\Delta_{i}\right|^{s}+\sum_{i=1}^{n}(1+M / 2 \eta)(2 \eta)^{s} \\
& \leq 2 \delta+\sum_{\Pi} \operatorname{osc}\left(f, \Delta_{i}\right)\left|\Delta_{i}\right|^{s-1} .
\end{aligned}
$$

By letting $\delta \rightarrow 0$, the inequality follows.

Conversely let $\delta>0$, and let $\mathscr{C}$ be an open cover of $[0,1]$. By the compactness of $[0,1]$ we can assume that $\mathscr{C}$ is finite, it is easy to construct a partition $\Pi$ from $\mathscr{C}$ such that

Applying (2.1) we obtain

$$
\sum_{\Pi} q_{\Delta_{i}^{\circ}}\left|\Delta_{i}\right|^{s} \leq \sum_{I \in \mathscr{C}} q_{I}|I|^{s} .
$$

$$
\sum_{\Pi} \operatorname{osc}\left(f, \Delta_{i}\right)\left|\Delta_{i}\right|^{s-1} \leq \sum_{I \in \mathscr{C}} q_{I}|I|^{s} .
$$

By taking the infimum the lemma follows. 
In the definition of $\mathscr{K}^{s}$, if we replace the cover $\mathscr{C}$ of open intervals and the corresponding open squares by nonoverlapping half open dyadic intervals and dyadic squares respectively, we obtain another measure $\mathscr{K}^{\prime s}$ (see [4, pp. 64-65]), and we have

Proposition 2.3. For any subset $E$ of $\Gamma_{f}, \mathscr{K}^{s}(E) \leq \mathscr{K}^{\prime s}(E) \leq c \mathscr{K}^{s}(E)$, for some constant $c$ independent of $f$ and $E$.

Analogous to the Hausdorff dimension we define the dimension index in terms of $\mathscr{K}^{s}$ by

$$
\mathscr{K}-\operatorname{dim} \Gamma_{f}=\inf \left\{s>0: \mathscr{K}^{s}\left(\Gamma_{f}\right)=0\right\} .
$$

It is easy to show that it also equals $\sup \left\{s>0: \mathscr{K}^{s}\left(\Gamma_{f}\right)=\infty\right\}$, and by Proposition $2.1,1 \leq \mathscr{K}-\operatorname{dim} \Gamma_{f} \leq 2$.

The following simple lemma gives a useful criterion for the lower bound of $\mathscr{K}-\operatorname{dim} \Gamma_{f}$, in particular when $f \in \operatorname{Lip}(\alpha)$. It will be used to study the Weierstrass type functions in $\S 4$.

Lemma 2.4. Let $0 \leq \alpha \leq 1$. Suppose that there exists a subset $A$ of $[0,1]$ such that $|A|>0$, and $\underline{\lim }_{\left|I_{x}\right| \rightarrow 0} \operatorname{osc}\left(f, I_{x}\right) /\left|I_{x}\right|^{\alpha}>0$, for all $x \in A$, then $\mathscr{K}^{2-\alpha}\left(\Gamma_{f}\right)>0$, and hence $\mathscr{K}-\operatorname{dim} \Gamma_{f} \geq 2-\alpha$.

Proof. The hypothesis implies that there exist $\eta>0$ and $c>0$ such that

$$
\left|\left\{x \in[0,1]: \inf _{\left|I_{x}\right|<\eta} \operatorname{osc}\left(f, I_{x}\right) /\left|I_{x}\right|^{\alpha}>c\right\}\right|>0 .
$$

Let $E$ be the subset in (2.3). For any open cover $\mathscr{C}$ of $[0,1]$, let $\mathscr{C}^{\prime}$ be the collection of all those $I$ in $\mathscr{C}$ intersecting $E$. Then, using $\delta<\eta$, we have, by (2.1),

$$
\mathscr{K}^{2-\alpha}\left(\Gamma_{f}\right) \geq \lim _{\delta \rightarrow 0^{+}} \inf _{|\mathscr{C}|<\delta} \sum_{I \in \mathscr{C}^{\prime}} c|I| \geq c|E|,
$$

proving the lemma.

\section{COMPARISON WITH THE OTHER DEFINITIONS}

Let $E$ be a subset in $\mathbb{R}^{2}$, and let $M_{n}(E)$ be the smallest number of open balls of radius $1 / n$ needed to cover $E$, define the lower box dimension by

$$
\delta(E)=\frac{\lim }{n} \frac{\log M_{n}(E)}{\log n},
$$

and the upper box dimension $\Delta(E)$ by replacing $\varliminf_{n}$ with $\overline{\lim }_{n}$. It is obvious that $\delta(E) \leq \Delta(E)$, and if the equality holds then the common value is called the box dimension of $E$.

Theorem 3.1. Let $f$ be a continuous function on $[0,1]$, then $\mathscr{H}-\operatorname{dim} \Gamma_{f} \leq \mathscr{K}$ $\operatorname{dim} \Gamma_{f} \leq \delta\left(\Gamma_{f}\right)$.

Proof. It suffices to prove the second inequality. It is known that the open balls in the definition of $M_{n}(E)$ can be replaced by dyadic squares of equal size $2^{-n}$. Also note that the $x$-projections of these squares form a cover $\mathscr{C}$ of $[0,1]$ of 
dyadic intervals of equal size. For any $s>\delta\left(\Gamma_{f}\right)$, then $\underline{\lim }_{n \rightarrow \infty} M_{2^{n}}\left(\Gamma_{f}\right) \cdot 2^{-n s} \leq$ 1 . It follows that

$$
\mathscr{K}^{\prime s}\left(\Gamma_{f}\right) \leq \varliminf_{n \rightarrow \infty} \sum_{I \in \mathscr{C}} q_{I}|I|^{s}=\varliminf_{n \rightarrow \infty} M_{2^{n}}\left(\Gamma_{f}\right) \cdot 2^{-n s} \leq 1 .
$$

Using Proposition 2.3 we see that $\mathscr{K}-\operatorname{dim} \Gamma_{f} \leq s$, and the proof is completed by letting $s \rightarrow \delta\left(\Gamma_{f}\right)$.

One of the shortcomings of the lower (and upper) box dimension is that $\delta(E)=\delta(\bar{E})$, where $\bar{E}$ is the closure of $E$. To eliminate this one may define

$$
\hat{\delta}(E)=\inf \left\{\sup \delta\left(E_{i}\right): E \subseteq \bigcup E_{i}\right\},
$$

and define $\hat{\Delta}$ similarly. Then $\hat{\delta} \leq \delta$ and $\mathscr{H}-\operatorname{dim} \leq \hat{\delta} \leq \hat{\Delta} \leq \Delta$. Note that $\hat{\Delta}$ is also equivalent to the packing dimension recently introduced by Taylor and Tricot (see [14, 15 and 16]).

For any interval $I$ of $[0,1]$ let $f_{I}$ be the restriction of a function $f$ on $I$. In comparison with $\mathscr{K}$-dim we have

Theorem 3.2. Let $f$ be a continuous function on $[0,1]$. If the values of $\mathscr{K}$ $\operatorname{dim} \Gamma_{f_{I}}$ are equal for all open intervals $I$ of $[0,1]$, then $\mathscr{K}-\operatorname{dim} \Gamma_{f} \leq \hat{\delta}\left(\Gamma_{f}\right)$.

Proof. By using the relation $\delta(E)=\delta(\bar{E})$, it is easy to verify that $\hat{\delta}\left(\Gamma_{f}\right)$ also equals to $\inf \left\{\sup _{i} \delta\left(\bar{E}_{i}\right): \Gamma_{f} \subseteq \bigcup \bar{E}_{i}, \bar{E}_{i} \subseteq \Gamma_{f}\right\}$. For arbitrary sequence of $\left\{\bar{E}_{i}\right\}$ in $\mathbb{R}^{2}$ whose union covering $\Gamma_{f}$, by the Baire's theorem, there is at least one of them with $x$-projection containing an interval $I$. The hypothesis and Theorem 3.1 (still valid with $\Gamma_{f}$ replaced by $\Gamma_{f_{l}}$ ) imply that $\sup \delta\left(\bar{E}_{i}\right) \geq \delta\left(\Gamma_{f_{l}}\right) \geq \mathscr{K}$ $\operatorname{dim} \Gamma_{f_{I}}=\mathscr{K}-\operatorname{dim} \Gamma_{f}$. This completes the proof since $\left\{\bar{E}_{i}\right\}$ is arbitrary.

For $1 \leq p \leq \infty$, let

$$
|f|_{p}=\lim _{\delta \rightarrow 0^{+}} \inf _{\Pi \Pi<\delta} \sum_{\Pi} \operatorname{osc}\left(f, \Delta_{i}\right)\left|\Delta_{i}\right|^{1-1 / p},
$$

and let

$$
\|f\|_{p}=\lim _{\delta \rightarrow 0^{+}} \sup _{|\Pi|<\delta} \sum_{\Pi} \operatorname{osc}\left(f, \Delta_{i}\right)\left|\Delta_{i}\right|^{1-1 / p},
$$

then both $\|\left.\right|_{p}$ and \|\|$_{p}$ are seminorms. As already proved in Proposition 2.2, if $f$ is continuous and $1<p \leq \infty$, then $|f|_{p}=\mathscr{K}^{2-1 / p}\left(\Gamma_{f}\right)$. We use $\mathscr{V}^{p}$ and $V^{p}$ to denote the two classes of functions for which $|f|_{p}<\infty$ and $\|f\|_{p}<\infty$ respectively. It is clear that $V^{p} \subseteq \mathscr{V}^{p}$, and if $p_{1} \leq p_{2}$ then $\mathscr{V}^{p_{1}} \subseteq \mathscr{V}^{p_{2}}$ and $V^{p_{1}} \subseteq V^{p_{2}}$.

Proposition 3.3. For $1 \leq p \leq \infty$, let $f \in \mathscr{V}^{p}$, then

(i) $f$ is bounded; and

(ii) if $1<p<\infty$, then $f$ is continuous almost everywhere with respect to $\mathscr{H}^{1-t}$ for every $t<1 / p$.

Proof. (i) Suppose that $|f|_{p}<\infty$, then $\sum_{\Pi} \operatorname{osc}\left(f, \Delta_{i}\right)\left|\Delta_{i}\right|^{1-1 / p}=M<\infty$ for some fixed partition $\Pi=\left\{0=x_{0}<x_{1}<\cdots<x_{n}=1\right\}$. We have

$$
\sup |f(x)| \leq|f(0)|+n \cdot \sup _{i} \operatorname{osc}\left(f, \Delta_{i}\right) \leq|f(0)|+n \cdot M / \min \left|\Delta_{i}\right|^{1-1 / p}<\infty .
$$


To prove (ii), let $E=\{x \in[0,1]: f$ is not continuous at $x\}$, and suppose that $\mathscr{H}^{1-t}(E)>0$, where $t<1 / p$. There exist a subset $F$ of $E$ and $\eta>0$ so that $\mathscr{C}^{1-t}(F)>0$ and $\operatorname{osc}\left(f, I_{x}\right)>\eta$, for every $x \in F$ and any interval $I_{x}$ containing $x$.

Let $\Pi$ be any partition of $[0,1]$, and let $\Delta_{1}^{\prime}, \ldots, \Delta_{k}^{\prime}$ be all those subintervals of $\Pi$ whose union covers $F$, then

$$
\begin{aligned}
\sum_{\Pi} \operatorname{osc}\left(f, \Delta_{i}\right)\left|\Delta_{i}\right|^{1-1 / p} & \geq \sum_{i=1}^{k} \operatorname{osc}\left(f, \Delta_{i}^{\prime}\right)\left|\Delta_{i}^{\prime}\right|^{1-1 / p} \geq \eta \sum_{i=1}^{k}\left|\Delta_{i}^{\prime}\right|^{1-1 / p} \\
\geq \eta \mathscr{K}_{|\Pi|}^{1-1 / p}(F) & \rightarrow \eta \mathscr{H}^{1-1 / p}(F), \quad \text { when }|\Pi| \rightarrow 0 .
\end{aligned}
$$

Since $\mathscr{H}^{1-t}(F)>0$, thus $\mathscr{H}^{1-1 / p}(F)=\infty$, this implies that $|f|_{p}=\infty$, contradicting that $f \in \mathscr{V}^{p}$.

Corollary 3.4. $V^{\infty}=\mathscr{V}^{\infty}$ and is exactly the class of bounded functions.

Proof. In view of Proposition 3.3(i), we only need to see that every bounded function is in $V^{\infty}$, but this follows directly from the definition.

Proposition 3.5. For $1 \leq p \leq \infty, f \in V^{P}$ if and only if

$$
\lim _{\delta \rightarrow 0+} \sup _{|\Pi|<\delta} \sum_{\Pi}\left|f\left(x_{i}\right)-f\left(x_{i-1}\right)\right|\left|\Delta_{i}\right|^{1-1 / p}<\infty .
$$

In particular, $f \in V^{1}$ if and only if $f$ is of bounded variation.

Proof. It suffices to show that there is a constant $c$ such that

$$
c\|f\|_{p} \leq \lim _{\delta \rightarrow 0^{+}} \sup _{|\Pi|<\delta} \sum_{\Pi}\left|f\left(x_{i}\right)-f\left(x_{i-1}\right)\right|\left|\Delta_{i}\right|^{1-1 / p} .
$$

For any partition $\Pi=\left\{0=x_{0}<x_{1}<\cdots<x_{n}=1\right\}$, we can find two points $y_{i}$ and $z_{i}$ in each $\Delta_{i}$ so that $\left|f\left(z_{i}\right)-f\left(y_{i}\right)\right|>\operatorname{osc}\left(f, \Delta_{i}\right) / 3$, and that $\left|z_{i}-y_{i}\right|>\left|\Delta_{i}\right| / 3$. Thus

$$
\left|f\left(z_{i}\right)-f\left(y_{i}\right)\right|\left|z_{i}-y_{i}\right|^{1-1 / p}>\operatorname{osc}\left(f, \Delta_{i}\right)\left|\Delta_{i}\right|^{1-1 / p} / 3^{2-1 / p} .
$$

Form the new partition $\Pi^{\prime}$ which includes the points of $\Pi$ and of $\left\{y_{i}, z_{i}: i=\right.$ $1, \ldots, n\}$, then

$$
\begin{aligned}
\sum_{\Pi^{\prime}}\left|f\left(x_{i}\right)-f\left(x_{i-1}\right)\right|\left|\Delta_{i}\right|^{1-1 / p} & \geq \sum_{i=1}^{n}\left|f\left(z_{i}\right)-f\left(y_{i}\right)\right|\left|z_{i}-y_{i}\right|^{1-1 / p} \\
& \geq 3^{1 / p-2} \sum_{\Pi} \operatorname{osc}\left(f, \Delta_{i}\right)\left|\Delta_{i}\right|^{1-1 / p}
\end{aligned}
$$

proving (3.1).

For $1 \leq p<\infty$, let $B V^{p}=\left\{f: \sup \sum_{\Pi}\left|f\left(x_{i}\right)-f\left(x_{i-1}\right)\right|^{p}<\infty\right\}$ be the class of functions of bounded p-variation (see Wiener [17]), and let $\mathscr{B} \mathscr{V}^{p}=$ $\left\{f: \lim _{\delta \rightarrow 0^{+}} \inf _{|\Pi|<\delta} \sum_{\Pi}\left(\operatorname{osc}\left(f, \Delta_{i}\right)\right)^{p}<\infty\right\}$ be the class of functions of bounded weak p-variation (see Goffman and Loughlin [3]). 
Proposition 3.6. Let $1<p<\infty$, then $\operatorname{Lip}(1 / p) \subseteq B V^{p} \subseteq V^{p}$, and $\mathscr{B} \mathscr{V}^{p} \subseteq$ $\mathscr{V}^{p}$.

Proof. The proposition follows from the Hölder inequality that

$$
\begin{aligned}
\sum\left|f\left(x_{i}\right)-f\left(x_{i-1}\right)\right|\left|\Delta_{i}\right|^{1-1 / p} & \leq\left(\sum\left|f\left(x_{i}\right)-f\left(x_{i-1}\right)\right|^{p}\right)^{1 / p}\left(\sum\left|\Delta_{i}\right|\right)^{1-1 / p} \\
& =\left(\sum\left|f\left(x_{i}\right)-\left(x_{i-1}\right)\right|^{p}\right)^{1 / p} .
\end{aligned}
$$

From Proposition 2.2, we see that if $f$ is a continuous function then $\mathscr{K}$ $\operatorname{dim} \Gamma_{f}$ actually equals to $\inf \left\{2-1 / p:|f|_{p}=0\right\}$. We can also define an analog dimension index by

$$
K-\operatorname{dim} \Gamma_{f}=\inf \left\{2-1 / p:\|f\|_{p}=0\right\} .
$$

This dimension index dominates the previous ones in the following sense:

Proposition 3.7. Let $f$ be a continuous function on $[0,1]$, then $\Delta\left(\Gamma_{f}\right) \leq K$ $\operatorname{dim} \Gamma_{f}$.

Proposition 3.8. Under the assumption of Lemma 2.4, if $f \in V^{p}$, then $0<$ $\mathscr{K}^{2-1 / p}\left(\Gamma_{f}\right)<\infty$, and $\mathscr{K}-\operatorname{dim} \Gamma_{f}=K-\operatorname{dim} \Gamma_{f}=2-1 / p$.

\section{THE MAIN THEOREM}

For the rest of this paper we will fix $0<\alpha<\beta \leq 1$. Let $g(x)$ defined on $\mathbb{R}$ be a real-valued (Bohr) bounded almost periodic Lipschitz function of order $\beta$. For simplicity, assume that $g(0)=0,|g(x)-g(y)| \leq|x-y|^{\beta}$ for all $x, y \in \mathbb{R}$ and $\sup _{x \in \mathbb{R}}|g(x)| \leq 1$. Let $W(x), V(x)$, and $E\left(f, \varepsilon, I_{x}\right)$ be defined by (1.2), (1.3), and (1.4) respectively. $W$ is clearly an almost periodic function, but $V$ is not. Indeed this follows directly from the equation

$$
V\left(\lambda^{i} x\right)=\lambda^{\alpha i} V(x), \quad x \in \mathbb{R},
$$

for any integer $i$.

Theorem 4.1. The following statements are equivalent:

(i) $V \not \equiv 0$;

(ii) There exist positive numbers $\varepsilon, \eta$ and $c$ such that $\left|E\left(W, \varepsilon, I_{x}\right)\right| /\left|I_{x}\right| \geq c$ for every $x \in \mathbb{R}$ and every interval $I_{x}$ containing $x$ with $\left|I_{x}\right|<\eta$;

(iii) $0<\mathscr{K}^{2-\alpha}\left(\Gamma_{w}\right)<\infty$, in this case $\mathscr{K}-\operatorname{dim} \Gamma_{w}=2-\alpha$.

Moreover the above equivalence is still valid if $W$ in (ii) and (iii) is replaced by $V$.

Before proving the theorem we make some remarks.

Remark 1 . We cannot replace $V$ by $W$ in (i), this can be justified by the following example essentially due to Kaplan et al. [10]:

Let $r(x)$ be any bounded almost periodic Lipschitz function of order $\beta$ so that $\mathscr{K}-\operatorname{dim} \Gamma_{r}$ equals $2-\beta$ and $r(0)=0$. Then $g(x)=r(x)-\lambda^{-\alpha} r(\lambda x)$ is also such a function. A simple computation shows that $W(x)=\sum_{0}^{\infty} \lambda^{-\alpha i} g\left(\lambda^{i} x\right)=$ $r(x)$, thus $\mathscr{K}-\operatorname{dim} \Gamma_{W}=2-\beta$.

Remark 2. From Theorem 4.1, we can deduce that $W$ and $V$ satisfy the hypotheses of Theorem 3.2, thus $\hat{\delta}\left(\Gamma_{W}\right)=\hat{\Delta}\left(\Gamma_{W}\right)=2-\alpha$, and the same conclusion 
holds with $\Gamma_{W}$ replaced by $\Gamma_{V}$. This improves the corresponding results of Kaplan et al. [10] and of Rezakhanlou [13].

Remark 3. Statement (ii) of Theorem 4.1 is the conclusion of Hata's Theorem 2.1 in [6]. A comparison of his conditions and that $V \not \equiv 0$ will be given in Proposition 5.6.

We need a lemma to prove the theorem. A subset $D$ of $\mathbb{R}$ is relatively dense if there is a number $h>0$ such that every interval $[x, x+h], x \in \mathbb{R}$, contains at least one point of $D$. It is known that a continuous function $g$ is almost periodic if and only if for any $\varepsilon>0$, there is a relatively dense subset $D=D(\varepsilon)$ of $\mathbb{R}$ such that for every $\tau \in D$ we have $|g(x+\tau)-g(x)| \leq \varepsilon$, for all $x \in \mathbb{R}$ (see [2]).

Lemma 4.2. If there exist $y \in \mathbb{R}$ and $a>0$ such that $V(y)>3 a($ or $<-3 a)$, then there exist a relatively dense subset $D$ of $\mathbb{R}$, an integer $r$, and a positive number $\sigma$ such that

$$
\left(W\left(\lambda^{-n}(t+y)\right)-W\left(\lambda^{-n} t\right)\right) / \lambda^{-\alpha n}>a \quad(\text { or }<-a),
$$

for all $n>r$ and every $t \in \bigcup_{\tau \in D}(\tau-\sigma, \tau+\sigma)$.

Proof. Let $r$ be a fixed integer to be determined in the proof, and let $V_{r}(t)=$ $\sum_{-r}^{r} \lambda^{-\alpha i} g\left(\lambda^{i} t\right)$. By the hypothesis on $g, V_{r} \in \operatorname{Lip}(\beta)$ and $V_{r}$ is an almost periodic function. Let $D$ be the relatively dense subset of $\mathbb{R}$ such that for every $\tau \in D$,

$$
\left|V_{r}(t+\tau)-V_{r}(t)\right|<a / 4, \quad \text { for all } t \in \mathbb{R} .
$$

Since $V_{r}(0)=0$, hence $\left|V_{r}(\tau)\right|<a / 4$ for all $\tau \in D$. Let $M_{r}$ be the Lipschitz constant of $V_{r}$. Let $\sigma=\left(a / 4 M_{r}\right)^{1 / \beta}$, then for $n>r$, and $t \in(\tau-\sigma, \tau+\sigma)$, where $\tau \in D$, we have

$$
\begin{aligned}
& W\left(\lambda^{-n}(t+y)\right)-W\left(\lambda^{-n} t\right) \\
& =\left(\sum_{i=0}^{n-r-1}+\sum_{i=n-r}^{n+r}+\sum_{i=n+r+1}^{\infty}\right) \lambda^{-\alpha i}\left(g\left(\lambda^{i-n}(t+y)\right)-g\left(\lambda^{i-n} t\right)\right) \\
& =S_{1}+S_{2}+S_{3} .
\end{aligned}
$$

If $r$ is chosen so that

$$
V_{r}(y)>3 a,
$$

then

$$
\begin{aligned}
S_{2} & =\sum_{i=n-r}^{n+r} \lambda^{-\alpha i}\left(g\left(\lambda^{i-n}(t+y)\right)-g\left(\lambda^{i-n} t\right)\right) \\
& =\lambda^{-\alpha n} \sum_{-r}^{r} \lambda^{-\alpha i}\left(g\left(\lambda^{i}(t+y)\right)-g\left(\lambda^{i} t\right)\right) \\
& =\lambda^{-\alpha n}\left(V_{r}(t+y)-V_{r}(t)\right) \\
& =\lambda^{-\alpha n}\left\{V_{r}(y)-\left[V_{r}(y)-V_{r}(\tau+y)\right]-\left[V_{r}(\tau+y)-V_{r}(t+y)\right]\right. \\
& \left.\geq \lambda^{-\alpha n}\left[3 a-a / 4-2 M_{r}(t)-V_{r}(\tau)\right]-V_{r}(\tau)\right\} \\
& =2 a \lambda^{-\alpha n} .
\end{aligned}
$$


If $r$ satisfies

$$
|y|^{\beta} \lambda^{(\alpha-\beta) r} /\left(\lambda^{\beta-\alpha}-1\right)<a / 2,
$$

using the Lipschitz condition of $g(t)$ then

$$
\left|S_{1}\right| \leq \sum_{i=0}^{n-r-1} \lambda^{-\alpha i}\left|\lambda^{i-n} y\right|^{\beta} \leq a \lambda^{-\alpha n} / 2
$$

If further $r$ satisfies

$$
1 / \lambda^{\alpha r}\left(\lambda^{\alpha}-1\right)<a / 4
$$

then

$$
\left|S_{3}\right| \leq 2 \sum_{i=n+r+1}^{\infty} \lambda^{-\alpha i}<a \lambda^{-\alpha n} / 2
$$

The lemma follows from the following inequality:

$$
W\left(\lambda^{-n}(t+y)\right)-W\left(\lambda^{-n} t\right) \geq S_{2}-\left|S_{1}\right|-\left|S_{3}\right|>a \lambda^{-\alpha n},
$$

provided that $r$ satisfies (4.2), (4.3), and (4.5).

For $x \in \mathbb{R}$ and any subset $A$ of $\mathbb{R}$, let $x+A=\{x+a: a \in A\}$ and let $x A=\{x a: a \in A\}$.

Proof of Theorem 4.1. (i) $\Rightarrow$ (ii): Since $V \not \equiv 0$, we can find a $y \in \mathbb{R}$ and $a>0$ such that $|V(y)|>3 a$. Let $\sigma>0$, integer $r$, and the relatively dense $D$ be as in Lemma 4.2. Let $h>0$ be such that every interval $[x, x+h], x \in \mathbb{R}$, contains at least one point of $D$. Without loss of generality assume that $\sigma<h$ and $0<y \leq h$.

Set $\eta=\lambda^{-r} h$. For any $x \in \mathbb{R}$ and any interval $I_{x}$ containing $x$ with $\left|I_{x}\right|<\eta$, let $n$ be the least integer so that

$$
4 h \leq \lambda^{n}\left|I_{x}\right|
$$

then $n>r$, and we can find some $\tau \in D$ such that both $(\tau-\sigma, \tau+\sigma)$ and $y+(\tau-\sigma, \tau+\sigma)$ are contained in $\lambda^{n} I_{x}$. Pick any $t \in(\tau-\sigma, \tau+\sigma)$, it follows from Lemma 4.2 that

$$
\begin{aligned}
a & <\frac{\left|W\left(\lambda^{-n}(t+y)\right)-W\left(\lambda^{-n} t\right)\right|}{\lambda^{-n \alpha}} \\
& \leq \frac{\left|W\left(\lambda^{-n}(t+y)\right)-W(x)\right|}{\lambda^{-n \alpha}}+\frac{\left|W(x)-W\left(\lambda^{-n} t\right)\right|}{\lambda^{-n \alpha}} .
\end{aligned}
$$

Then

$$
\begin{aligned}
\max & \left\{\frac{\left|W\left(\lambda^{-n}(t+y)\right)-W(x)\right|}{\left|\lambda^{-n}(t+y)-x\right|^{\alpha}}, \frac{\left|W\left(\lambda^{-n} t\right)-W(x)\right|}{\left|\lambda^{-n} t-x\right|^{\alpha}}\right\} \\
& \geq \max \left\{\frac{\left|W\left(\lambda^{-n}(t+y)\right)-W(x)\right|}{\left|I_{x}\right|^{\alpha}}, \frac{\left|W\left(\lambda^{-n} t\right)-W(x)\right|}{\left|I_{x}\right|^{\alpha}}\right\} \\
& >a / 2\left(\lambda^{n}\left|I_{x}\right|\right)^{\alpha} \quad(\text { by (4.7)) } \\
& \geq \varepsilon, \quad(\text { by }(4.6))
\end{aligned}
$$

where $\varepsilon=a(4 \lambda h)^{\alpha} / 2$. Since $t \in(\tau-\sigma, \tau+\sigma)$ is arbitrary, the above estimate implies that $\left|E\left(W, \varepsilon, I_{x}\right)\right| \geq 2 \sigma \lambda^{-n}$. Again by (4.6) we obtain that $\left|E\left(W, \varepsilon, I_{x}\right)\right| /\left|I_{x}\right| \geq c$, where $c$ equals to $\sigma / 2 h \lambda$. This proves (ii). 
(ii) $\Rightarrow$ (iii): For any $x \in \mathbb{R}$ and every interval $I_{x}$ containing $x$ with $\left|I_{x}\right|$ small enough then

$$
\begin{aligned}
\operatorname{osc}\left(W, I_{x}\right) & \geq \sup _{s \in E}|W(s)-W(x)| \\
& \geq \varepsilon \sup _{s \in E}|s-x|^{\alpha} \geq \varepsilon\left(c\left|I_{x}\right| / 2\right)^{\alpha},
\end{aligned}
$$

where $E=E\left(W, \varepsilon, I_{x}\right)$. Hence $\underline{\lim }_{\left|I_{x}\right| \rightarrow 0} \operatorname{osc}\left(W, I_{x}\right) /\left|I_{x}\right|^{\alpha}>0$, for every $x \in \mathbb{R}$. Observe that $W \in \operatorname{Lip}(\alpha) \subseteq V^{1 / \alpha}$, thus (iii) follows from Proposition 3.8.

(iii) $\Rightarrow$ (i): Since $(W-V) \in \operatorname{Lip}(\beta)$, it follows from Proposition 3.8 that the $\mathscr{K}$-dimension of the graph of $(W-V)$ is less than $2-\beta$, hence $V \not \equiv 0$.

By using $(W-V) \in \operatorname{Lip}(\beta)$, it is easy to show that the theorem remains true with $W$ replaced by $V$. This completes the proof of Theorem 4.1.

Remark 4. Lemma 4.2 and Theorem 4.1 remain true if we relax the condition that $g(x) \in \operatorname{Lip}(\beta)$ to be

$$
\sup _{x \in \mathbb{R}}|g(x+h)-g(x)|=o\left(|h|^{\alpha} /(\log |h|)^{p}\right), \quad \text { as } h \rightarrow 0,
$$

for some $p>1$. All we need to adjust is the estimate of $S_{1}$ in (4.4):

$$
\left|S_{1}\right| \leq c \sum_{i=0}^{n-r-1} \lambda^{-\alpha i}\left|\lambda^{i-n} y\right|^{\alpha} /\left(\log \left|\lambda^{i-n} y\right|\right)^{p} \leq a \lambda^{-\alpha n} / 2,
$$

where $c$ is a positive number as small as we like.

We conclude this section by giving a simple criterion to guarantee that $V \not \equiv 0$.

Proposition 4.3. Let $g \in \operatorname{Lip}(\beta)$. Assume that there is a $c>0$ such that $G(x)=\int_{0}^{x} g(t) d t \geq 0$, for $0<x \leq c \leq \infty$, and $M=G(a)>0$, for some $a \in(0, c]$. Let $m=\inf \{G(x): x \in(a \lambda, \infty)\}$, if $M>(-m) /\left(\lambda^{\alpha+1}-1\right)$, then $V \not \equiv 0$.

Proof. Since $V(x)=\sum_{-\infty}^{\infty} \lambda^{-\alpha i} g\left(\lambda^{i} x\right)$ converges uniformly on compact subsets, integrating term by term gives

$$
\begin{aligned}
\int_{0}^{a} V(t) d t & =\left(\sum_{\infty}^{0}+\sum_{1}^{\infty}\right) \lambda^{-(\alpha+1) i} G\left(\lambda^{i} a\right) \\
& \geq G(a)+m \sum_{1}^{\infty} \lambda^{-(\alpha+1) i}=M+m /\left(\lambda^{\alpha+1}-1\right),
\end{aligned}
$$

proving the proposition.

The condition of Proposition 4.3 can be applied easily, e.g., to the classical cases: $g(x)=\sin x$, or $g(x)$ is of period 1 and equals to $1-|1-2 x|$ on [0, 1] (the corresponding $W$ is called the Takagi function). Actually in both cases we have $m \geq 0$.

\section{SiNGULARITIES OF THE WeIERSTRASS TYPE FUNCTIONS}

For any real-valued function of $\mathbb{R}$, we let

$$
\begin{aligned}
& D^{+} f(x)=\lim \sup _{h \rightarrow 0^{+}} \frac{f(x+h)-f(x)}{h}, \quad \text { and } \\
& D_{+} f(x)=\lim \inf _{h \rightarrow 0^{+}} \frac{f(x+h)-f(x)}{h}
\end{aligned}
$$


be the upper right derivative and the lower right derivate of $f$ at $x$. Similarly we can define the upper and lower left derivative of $f$ at $x$, denoted by $D^{-} f(x)$ and $D_{-} f(x)$. A point $x \in \mathbb{R}$ is called a knot point of a real-value function $f(x)$ provided that

$$
D^{+} f(x)=D^{-} f(x)=\infty, \quad \text { and } \quad D_{+} f(x)=D_{-} f(x)=-\infty .
$$

The set of knot points of $f(x)$ is denoted by $\operatorname{Knot}(f)$. Another type of singularity, in contrast to the knot point, is the notion of differential coefficient $\infty$ at $x$ defined by $D^{+} f(x)=D^{-} f(x)=D_{+} f(x)=D_{-} f(x)=\infty$. For $\varepsilon>0$, $\eta>0$ and $x \in \mathbb{R}$, let

$$
E_{x}^{p}(f, \varepsilon, \eta)=\left\{s \in(x, x+\eta):(f(s)-f(x)) /|s-x|^{\alpha}>\varepsilon\right\},
$$

and

$$
E_{x}^{n}(f, \varepsilon, \eta)=\left\{s \in(x, x+\eta):(f(s)-f(x)) /|s-x|^{\alpha}<-\varepsilon\right\} .
$$

Similarly define $E_{x}^{p}(f, \varepsilon,-\eta)$ and $E_{x}^{n}(f, \varepsilon,-\eta)$ with $(x, x+\eta)$ in $(5.1)$ and (5.2) replaced by $(x-\eta, x)$ respectively.

For the rest of the section, unless specified, $W$ and $V$ will be defined by (1.2) and (1.3).

Theorem 5.1. Suppose that $V \not \equiv 0$, then there exist positive constants $\varepsilon$ and $c$, and a dense $G_{\delta}$-subset $G$ of $\mathbb{R}$ with $|\mathbb{R} \backslash G|=0$ such that for every $x \in G$,

$$
\begin{aligned}
\varlimsup_{\eta \rightarrow 0+} \min \left\{\frac{\left|E_{x}^{p}(W, \varepsilon, \eta)\right|}{\eta}, \frac{\left|E_{x}^{n}(W, \varepsilon, \eta)\right|}{\eta},\right. \\
\left.\frac{\left|E_{x}^{p}(W, \varepsilon,-\eta)\right|}{\eta}, \frac{\left|E_{x}^{n}(W, \varepsilon,-\eta)\right|}{\eta}\right\} \geq c .
\end{aligned}
$$

In particular, $\operatorname{Knot}(W)$ contains a dense $G_{\delta}$-set, and $\mathbb{R} \backslash \operatorname{Knot}(W)$ is of Lebesgue measure zero. Moreover, the theorem remains true with $W$ replaced by $V$.

Remark 1. The above theorem (without the dense $G_{\delta}$ part) is stated in Hata [6, Theorem 3.1] with some other assumptions (see the remark before Proposition 5.6). It seems that there is a gap in his proof (p. 77, line 11), and his conclusion in (5.3) can only be stated as the minimum of the limit supremum of the four terms greater than $c$ instead.

Remark 2. By modifying the example in Remark 1 of the last section, we see that $V$ cannot be replaced by $W$ in the assumption.

We need the following two lemmas to obtain an estimation analogous to (4.8) in the proof of Theorem 4.1.

Lemma 5.2. Given that $\lambda>1$, and $\sigma>0$. Let $D$ be any relatively dense subset of $\mathbb{R}$. Then there exists a dense $G_{\delta}$-subset $G$ of $\mathbb{R}$ with $|\mathbb{R} \backslash G|=0$ satisfying: for every $x \in G, z \in \mathbb{R}$, there exists infinitely many positive integers $n$ such that

$$
\lambda^{n} x \in z+\bigcup_{\tau \in D}(\tau-\sigma, \tau+\sigma) .
$$

Proof. Without loss of generality assume that $z=0$. Let $I$ be any open interval with $|I|<\infty$, let $h>0$ be such that every interval $(x, x+h)$, 
$x \in \mathbb{R}$, contains at least one point of $D$ and that $2 \sigma \leq h$, and let $U=$ $\bigcup_{\tau \in D}(\tau-\sigma, \tau+\sigma)$. For each positive integer $p$, let $B_{p}=\left(\lambda^{p} I\right) \cap U$, and let $A_{p}=\lambda^{-p} B_{p}$. Then it is easy to check that for each $n \geq 1, \bigcup_{p \geq n} A_{p}$ is open and dense in $I$. Let $A=\bigcap_{n \geq 1} \bigcup_{p \geq n} A_{p}$, then $A$ is a dense $G_{\delta}$-subset of $I$, and each $x \in A$ will satisfy (5.4).

We will complete the proof by showing that $|A|=|I|$, or equivalently, $\left|\bigcup_{p \geq n} A_{p}\right|=|I|$ for arbitrary $n$. Suppose this is not true, let $G_{n}=\bigcup_{p \geq n} A_{p}$, and assume that $\left|I \backslash G_{n}\right|=\eta>0$. Then we can find a sequence of disjoint intervals $\left\{I_{i}\right\}_{1}^{\infty}$ of the form $[a, b) \cap I$ such that $J=\bigcup_{i \geq 1} I_{i}$ covers $I \backslash G_{n}$ and that

$$
|J| \leq \eta+\varepsilon,
$$

where $\varepsilon$ is a small positive number which will be determined later.

For each $i$, choose an integer $k_{i} \geq n$ such that

$$
\lambda^{k_{i}}\left|I_{i}\right|>h+2 \sigma \geq \lambda^{k_{i}-1}\left|I_{i}\right| \text {. }
$$

This can always be done by reducing the size of $I_{i}$ if necessary. Let $B_{i}^{\prime}=$ $\left(\lambda^{k_{i}} I_{i}\right) \cap U$, and let $C_{k_{i}}=\lambda^{-k_{i}} B_{i}^{\prime}$. Since $\lambda^{k_{i}} I_{i}$ contains at least one interval $(\tau-\sigma, \tau+\sigma)$ for some $\tau \in D$ by (5.6), hence

$$
\left|C_{k_{i}}\right| /\left|I_{i}\right| \geq\left(\lambda^{-k_{i}} 2 \sigma\right) /\left|I_{i}\right| \geq\left(\lambda^{-k_{i}} 2 \sigma\right) / \lambda^{-\left(k_{i}-1\right)}(h+2 \sigma)=c,
$$

where $c=2 \sigma / \lambda(h+2 \sigma)$. By the definition of $A_{p}$ we see that $C_{k_{i}} \subseteq A_{k_{i}} \subseteq G_{n}$. Observe that $C_{k_{i}} \subseteq I_{i}$, the above estimate yields

$$
\left|J \cap G_{n}\right|=\sum_{i=1}^{\infty}\left|I_{i} \cap G_{n}\right| \geq \sum_{1}^{\infty} c\left|I_{i}\right| \geq c \eta .
$$

Since $\left|J \backslash G_{n}\right|=\left|I \backslash G_{n}\right|=\eta$, thus $|J|=\left|J \cap G_{n}\right|+\left|J \backslash G_{n}\right| \geq \eta+c \eta$, this contradicts (5.5) if we choose $\varepsilon<c \eta$, and the proof is completed.

If $V \not \equiv 0$, by Theorem 4.1 we see that $V$ is nowhere monotone, therefore we can find a positive number $a>0$, and $z, x_{i}$, and $y_{i}$ in $[0, \infty), i=1,2$, such that $x_{1}<z<x_{2}, y_{1}<z<y_{2}$, and they satisfy

$$
V\left(x_{i}\right)-V(z)>3 a, \quad \text { and } \quad V\left(y_{i}\right)-V(z)<-3 a, \quad i=1,2 .
$$

By an obvious modification of the proof of Lemma 4.2 we can prove the following

Lemma 5.3. If $V \not \equiv 0$, then there exist $z, x_{i}$, and $y_{i}, i=1,2$, as above, and there also exist a relatively dense subset $D$ of $\mathbb{R}, a>0, \sigma>0$ and an integer $r$ such that

$$
\begin{aligned}
& \left(W\left(\lambda^{-n}(t+x)\right)-W\left(\lambda^{-n} t\right)\right) / \lambda^{-\alpha n}>a, \quad \text { and } \\
& \left(W\left(\lambda^{-n}(t+y)\right)-W\left(\lambda^{-n} t\right)\right) / \lambda^{-\alpha n}<-a,
\end{aligned}
$$

for all $n>r, t \in z+\bigcup_{\tau \in D}(\tau-\sigma, \tau+\sigma), x \in x_{i}-z+(-\sigma, \sigma)$ and $y \in$ $y_{i}-z+(-\sigma, \sigma), i=1,2$.

Proof of Theorem 5.1. We use the same notations as in Lemma 5.3. Let $h>0$ be such that every interval $(x, x+h), x \in \mathbb{R}$, contains at least one point of $D$ and that $x_{2}<h, y_{2}<h$ and $\sigma \leq \min \left\{\left|x_{i}-z\right|,\left|y_{i}-z\right|: i=1,2\right\}$. By 
Lemma 5.2, there exists a dense $G_{\sigma}$-subset $G$ of $\mathbb{R}$ with $|\mathbb{R} \backslash G|=0$ satisfying for every $x \in G$, there exist infinitely many positive integers $n$ such that

$$
\lambda^{n} x \in z+\bigcup_{\tau \in D}(\tau-\sigma, \tau+\sigma) .
$$

For each $x \in G$, we can find $n_{i}=n_{i}(x)>r$ and $\tau_{i} \in D, i=1,2, \ldots$, such that

$$
\lambda^{R_{i}} x \in z+\left(\tau_{i}-\sigma, \tau_{i}+\sigma\right) .
$$

Let $\eta_{i}=3 h \lambda^{-n_{i}}$. Then $\lambda^{n_{i}} x+\left(x_{2}-z\right)+(-\sigma, \sigma)$ is contained in the interval $\lambda^{n_{i}}\left(x, x+\eta_{i}\right)$. Hence for arbitrary $\bar{x} \in x_{2}-z+(-\sigma, \sigma), y=\lambda^{-n_{i}} \bar{x}$, we have $x+y \in\left(x, x+\eta_{i}\right)$. Thus (5.7) and Lemma 5.3 imply

$$
(W(x+y)-W(x)) / \lambda^{-n_{i} \alpha}>a .
$$

Hence

$$
(W(x+y)-W(x)) / y^{\alpha}=(W(x+y)-W(x)) /\left(\lambda^{-n_{i}} \bar{x}\right)^{\alpha}>\varepsilon,
$$

where $\varepsilon=a /(3 h)^{\alpha}$. This implies that $\left|E_{x}^{p}\left(W, \varepsilon, \eta_{i}\right)\right| \geq 2 \sigma \lambda^{-n_{i}}$. Therefore $\left|E_{x}^{p}\left(W, \varepsilon, \eta_{i}\right)\right| / \eta_{i} \geq c$, where $c=2 \sigma / 3 h$.

Similarly we can prove that $\left|E_{x}^{n}\left(W, \varepsilon, \eta_{i}\right)\right| / \eta_{i},\left|E_{x}^{p}\left(W, \varepsilon,-\eta_{i}\right)\right| / \eta_{i}$, and $\left|E_{x}^{n}\left(W, \varepsilon,-\eta_{i}\right)\right| / \eta_{i}$ are greater than $c$. Thus the theorem follows by letting $i \rightarrow \infty$.

It is trivial to see that for those $x$ where local maximum or local minimum occurs, then $x$ cannot be knot points. Furthermore, as pointed out by Hardy [5, Theorem 2.71] that if $\lambda^{-\alpha}(\lambda+1)<2$, then $W$ defined by (1.1) has the differential coefficient $\infty$ at the origin, and hence the origin is not a knot point. In general, we have

Proposition 5.4. If $V \not \equiv 0$, then $W$ (defined by (1.2)) has the differential coefficient $\infty$ at the origin if and only if $V(x)>0$ for all $x>0$, and $V(x)<0$ for all $x<0$.

Proof. Assume that $V(x)>0$ for all $x>0$ and $V(x)<0$ for all $x<0$. Applying Lemma 4.2, and noting that 0 can be selected into the relatively dense subset $D$, we have for large $n$

$$
\begin{gathered}
W\left(\lambda^{-n} y\right) / \lambda^{-\alpha n}>0, \quad \text { for all } y>0 ; \text { and } \\
W\left(\lambda^{-n} y\right) / \lambda^{-\alpha n}<0, \quad \text { for all } y<0 .
\end{gathered}
$$

Hence $W$ has the differential coefficient $\infty$ at the origin.

Conversely, if $V(y) \leq 0$ for some $y>0$, then by Lemma 4.2 and the equation (4.1) we obtain that

$$
\varliminf_{x \rightarrow 0^{+}} W(x) / x=-\infty \text { or } 0,
$$

this contradicts to $\varliminf_{x \rightarrow 0^{+}} W(x) / x=\infty$. Hence $V(x)>0$ for all $x>0$.

Corollary 5.5 (Hardy). If $\lambda^{-\alpha}(\lambda+1) \leq 2$, then $W$ defined by (1.1) has the differential coefficient $\infty$ at the origin. 
Proof. For any $x>0$, choose an integer $r$ such that $\lambda^{r-1} x \leq 1 / 2<\lambda^{r} x$. By using the inequality $\sin x / x \geq 2 / \pi$, for $0<x \leq \pi / 2$, we obtain

$$
\begin{aligned}
V(x) & =\sum_{-\infty}^{r-1} \lambda^{-\alpha i} \sin \left(\lambda^{i} \pi x\right)+\sum_{r}^{\infty} \lambda^{-\alpha i} \sin \left(\lambda^{i} \pi x\right) \\
& \geq \frac{2}{\pi} \sum_{-\infty}^{r-1} \lambda^{-\alpha i}\left(\lambda^{i} \pi x\right)-\sum_{r}^{\infty} \lambda^{-\alpha i} \\
& =\left[2 x \lambda^{r} /\left(\lambda^{1-\alpha}-1\right)-1 /\left(1-\lambda^{-\alpha}\right)\right] \lambda^{-\alpha r} \\
& >\left[1 /\left(\lambda^{1-\alpha}-1\right)-1 /\left(1-\lambda^{-\alpha}\right)\right] \lambda^{-\alpha r} \geq 0,
\end{aligned}
$$

with the given condition. Since $V(x)$ is an odd function, we thus have $V(x)<$ 0 for all $x<0$, proving the corollary.

To conclude this section we will give a discussion of the condition $V \not \equiv 0$ and the conditions of the Fourier coefficients of $W$ used in Hata [6] and Kaplan et al. [10]. For any bounded real-valued almost periodic function $f$ and $\xi \in \mathbb{R}$, let

$$
\hat{f}(\xi)=\lim _{T \rightarrow \infty} \frac{1}{T} \int_{0}^{T} f(x) e^{-i \xi x} d x
$$

to be the Fourier coefficient of $f$, then

$$
g(x) \sim \sum_{\xi} \hat{g}(\xi) e^{i \xi x} .
$$

It is known that the set $S=\{\xi: \hat{g}(\xi) \neq 0\}$ is at most countable, and the Fourier coefficient $\widehat{W}(\xi)$ of $W$ is given by $\widehat{W}(\xi)=\sum_{0}^{\infty} \lambda^{-\alpha i} \hat{g}\left(\lambda^{-i} \xi\right)$; here we are summing only those $i$ for which $\lambda^{-i} \xi \in S$. Hata [6, Theorem 2.1] proved (ii) of Theorem 4.1 by assuming the conditions $g \in \operatorname{Lip}(\beta)$ and

$$
\varlimsup_{\xi \rightarrow \infty}\left|\xi^{\alpha} \widehat{W}(\xi)\right|>0
$$

In his theorem of knot points of $W$, [6, Theorem 3.1], he made use of the condition (5.8) and

$$
\sum_{\xi \geq 0} \xi^{\alpha}|\hat{g}(\xi)|<\infty
$$

(condition (5.9) has also been used in Kaplan et al. [10]). To compare these conditions with the condition that $V \not \equiv 0$, we have

Proposition 5.6. Let $V_{k}(x)=\sum_{-k}^{\infty} \lambda^{-\alpha i} g\left(\lambda^{i} x\right)$, consider the following statements:

(i) $\overline{\lim }_{k \rightarrow \infty}\left|\widehat{V}_{k}(\xi)\right|>0$, for some $\xi \in \mathbb{R}$;

(ii) $\varlimsup_{\lim _{\xi \rightarrow \infty}}\left|\xi^{\alpha} \widehat{W}(\xi)\right|>0$;

(iii) $V \not \equiv 0$.

If $g \in \operatorname{Lip}(\beta)$ with $g(0)=0$, then (i) $\Leftrightarrow$ (ii) $\Rightarrow$ (iii). If in addition assume that $g$ satisfies (5.9), then these three statements are equivalent. 
Proof. (i) $\Rightarrow$ (ii). (Without assuming that $g \in \operatorname{Lip}(\beta)$.) Let $\xi \in \mathbb{R}$ so that $\varlimsup_{k \rightarrow \infty}\left|\widehat{V}_{k}(\xi)\right|>0$, and let $\xi_{k}=\lambda^{k} \xi$, then

$$
\begin{aligned}
\varlimsup_{k \rightarrow \infty}\left|\xi_{k}^{\alpha} \widehat{W}\left(\xi_{k}\right)\right| & =|\xi|^{\alpha} \varlimsup_{k \rightarrow \infty}\left|\sum_{i=0}^{\infty} \lambda^{(k-i) \alpha} \hat{g}\left(\lambda^{k-i} \xi\right)\right| \\
& =|\xi|^{\alpha} \varlimsup_{k \rightarrow \infty}\left|\sum_{i=-k}^{\infty} \lambda^{-\alpha i} \hat{g}\left(\lambda^{-i} \xi\right)\right| \\
& =|\xi|^{\alpha} \varlimsup_{k \rightarrow \infty}\left|\widehat{V}_{k}(\xi)\right|>0 .
\end{aligned}
$$

(ii) $\Rightarrow$ (i). We apply a method of Hata [6, p. 65]. Let $U_{k}(x)=V_{k}(x)-W(x)$, then there exists $M>0$ such that $\sup _{k}\left|U_{k}(x)-U_{k}(y)\right| \leq M|x-y|^{\beta}$. Now for any integer $n$ and $\xi>0$,

$$
\begin{aligned}
\left|\widehat{U}_{k}(\xi)\right| & =\lim _{n \rightarrow \infty} \frac{\xi}{2 \pi n}\left|\int_{0}^{2 \pi n / \xi} U_{k}(x) e^{-i \xi x} d x\right| \\
& =\lim _{n \rightarrow \infty} \frac{\xi}{2 \pi n}\left|\sum_{k=0}^{n-1} \int_{0}^{2 \pi / \xi}\left[U_{k}\left(\frac{2 k \pi}{\xi}+x\right)-U_{k}\left(\frac{2 k \pi}{\xi}\right)\right] e^{-i \xi x} d x\right| \\
& \leq \lim _{n \rightarrow \infty} \frac{\xi}{2 \pi n}\left|\sum_{k=0}^{n-1} \int_{0}^{2 \pi / \xi} M x^{\beta} d x\right| \\
& =c \xi^{-\beta}
\end{aligned}
$$

where $c=M(2 \pi)^{\beta} /(1+\beta)$. Note that $\widehat{U}_{k}(\xi)=\widehat{V}_{k}(\xi)-\widehat{W}(\xi)$, hence (ii) implies that for all sufficiently large $\xi, \varlimsup_{k \rightarrow \infty}\left|\widehat{V}_{k}(\xi)\right|>0$.

To see the relation between (ii) and (iii), observe that if the condition $g \in$ $\operatorname{Lip}(\beta)$ (respectively $g$ satisfies (5.9)) is assumed, the (ii) implies (respectively is equivalent to) the second statement of Theorem 4.1 (see [6, Theorem 2.1 and Theorem 2.4] respectively), but the latter is equivalent to (iii).

Remark. The equivalence of (i) and (iii) was also proved by Kaplan et al. [10, Proposition 2.2] under the conditions that $g$ is a smooth function and satisfies (5.9).

\section{REFERENCES}

1. A. S. Besicovitch and H. D. Ursell, Sets of fractional dimensions (v): On dimensional numbers of some continuous curves, J. London Math. Soc. 12 (1937), 18-25.

2. H. Bohr, Almost periodic functions, Chelsea, 1947.

3. C. Goffman and J. J. Loughlin, Strong and weak $\Phi$-variation of Brownian motion, Indiana Univ. Math. J. 22 (1972), 135-138.

4. K. J. Falconer, The geometry of fractal sets, Cambridge Univ. Press, 1985.

5. G. H. Hardy, Weierstrass' non-differentiable function, Trans. Amer. Math. Soc. 17 (1916), 301-325.

6. M. Hata, Singularities of the Weierstrass type functions, J. Analyse Math. 51 (1988), 62-90.

7. T. Y. Hu and K. S. Lau, The sum of Rademacher functions and Hausdorff dimension, Math. Proc. Cambridge Philos. Soc. 108 (1990), 97-103.

8. _ The sum of Rademacher functions and Hausdorff dimension. II (preprint). 
9. J. P. Kahane and R. Salem, Sur la convolution d'une infinite de distributions de Bernoulli, Colloq. Math. 6 (1958), 193-202.

10. J. L. Kaplan, J. Mallet-Paret, and J. A. Yorke, The Lyapunov dimension of a nowhere differentiable attracting torus, Ergodic Theory Dynamical Systems 4 (1984), 261-281.

11. R. D. Mauldin and S. C. Williams, On the Hausdorff dimension of some graphs, Trans. Amer. Math. Soc. 298 (1986), 793-803.

12. F. Przytycki and M. Urbanski, On the Hausdorff dimension of some fractal sets, Studia Math. 93 (1989), 155-186.

13. F. Rezakhanlou, The packing measure of the graphs and level sets of certain continuous functions, Math. Proc. Cambridge Philos. Soc. 104 (1988), 347-360.

14. S. J. Taylor, The measure theory of random fractals, Math. Proc. Cambridge Philos. Soc. 100 (1986), 383-406.

15. S. J. Taylor and C. Tricot, Packing measure, and its evaluation for a Brownian path, Trans. Amer. Math. Soc. 288 (1985), 679-699.

16. C. Tricot, Two definitions of fractional dimension, Math. Proc. Cambridge Philos. Soc. 91 (1982), 57-74.

17. N. Wiener, The quadratic variation of a function and its Fourier coefficients, J. Mass. Inst. Tech. 3 (1924), 73-94.

Department of Mathematics, University of Wisconsin-Green Bay, Green Bay, WisCONSIN 54311

Department of Mathematics and Statistics, University of Pittsburgh, Pittsburgh, Pennsylvania 15260 